\title{
PSDRA: Priority selection delivery routing algorithm in opportunity network
}

\author{
Jia WU, Zhi-gang CHEN \\ School of Software, Central South University \\ Changsha 410075, China \\ Jiawu5110@163.com
}

\begin{abstract}
The randomness of the opportunistic network and the disconnection of transmission area results in many difficulties in information transmission. In this paper, a new routing algorithm is called priority selection delivery routing algorithm (PSDRA). It has been designed through selecting the optimal node to make the next hop. This algorithm layers the communication nodes within opportunistic communication time and selects the optimal node to deliver the information, which helps the information to be delivered effectively and to reduce the active nodes' energy consumption in opportunistic communication area. PSDRA improve the delivery ratio, reduce energy consume and cache time. Compared with Direct Delivery routing algorithm, Epidemic routing algorithm, Spray and Wait routing algorithm in opportunistic network, PSDAR improves the performance of the routing algorithm.
\end{abstract}

Key words: opportunistic network; routing algorithm; node energy consumption; delivery ratio; cache time;

\section{INTRODUCTION}

In recent years emergence of a new kind of multi-hop wireless network known as opportunistic networks. It originate from DTN [1]. The key that distinguishing the feature of opportunistic networks and DTN from Ad Hoc Network[2] is that there never be an end-to-end path, but the union of network may present an end-to-end path at snapshots over time. MANET routing algorithm[3][4] drop packets in such situations and thus are insufficient. DTN include military communications, inter-planetary networks, networks in under-developed areas[5].

In opportunistic networks, the traditional algorithms paradigm of internet and Ad hoc[3], in which routing algorithms are computed based on topological information, but are not adequate anymore. The First approach to routing which is in opportunistic networks is variation of controlled flooding. All messages are flooded with limited by TimeTo-Live, and then delivered messages to the destination. It gets in touch with node that received the message during the flood.

The routing algorithms can forward via messages destine to people living in the same place, or nearby. Exploiting information is somewhat embedded in works on the topic. For example, Epidemic [6][7], exploit the frequency of contacts between nodes. Moby Space [8] and MV [9] exploit information about nodes' mobility patterns and place nodes that are used to visit. Then these data are become as context information.

\section{Related work}

In recent years, many studies have been made upon the replication-based routing algorithm transmission technology, such as Epidemic routing algorithm[10]. With the rapid increase of nodes, the actual net nodes cache resources wound become more and more insufficient. Thus, adding the transmission overhead and energy consumption, as a result, the performance of the replication-based transmission mechanism declines. Some defects exist in those improved the replication-based routing algorithm. Typical examples to illustrate is: MaxProp[11] routing algorithm, though reduces the overhead, makes it difficult to set up the expiry date of information properly; Spray and Wait [12] routing algorithm decreases the transmission of the useless information, thus reducing the overhead. However, the disagreement between the estimation of the historical information and the actual conditions may influence its delivery ratio and energy consumption. There are also other algorithms, Direct Delivery routing algorithm increases the energy consumption because of direct transmission; Spray and Wait algorithm may consume more energy for the reason of great deal of spraying during its judging of the first transmission.

Having found the defects of all those algorithms, it is written this in order to explore an algorithm that consumes less energy, transmits information more efficiently and decreases the time of cache appropriately. This kind of algorithm is designed on the basis of the next hop model which can be described as follows: to reduce the consumption of energy, the time of cache and to improve the transmission rate by choosing the optimized nodes, thus to improved the performance of opportunistic networks.

\section{Algorithm Design}

Opportunistic network is featured with nodes' mobility, which makes the whole network topology change constantly. How to make the node chose a better communication node in its communication area during its moving is an important problem. In the actual application, the subject of study is the node participating in communication in the area. The node that participating in communication is more active than others, which means that the information could be delivered in longer time, while the node that does not participate in communication probably be isolated when it participates in delivery. If those nodes do not participate in the delivery, the information is lost or unable to be delivered. Therefore, 
the node participating in communication is of great importance in the design of algorithm.

Algorithm is designed by these conditions in opportunistic networks:

1. Dividing the opportunistic network with strong randomness into several connected sub-networks in one period of time.

2. Studying the communication of opportunistic network during a period of time.

3. The subject of study is the node having chance to participate in opportunistic networks.

4. That the moving speed of nodes is slower than the information transmission speed could reflect life situation truly.

\subsection{Model research}

The typical structure of opportunistic network is that the topology structure of random network form different connected area in a period of time. In figure 1, during the time of $\mathrm{T}$, the opportunistic network is divided into two connected areas. In these two areas, nodes could deliver information, but the information could not be transmitted out of these areas. Only when the node carrying information moves could the information be transmitted out of these areas.

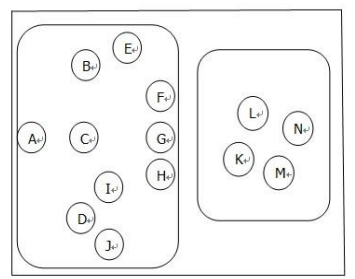

Figure 1: The structure of opportunistic network

According to the structure picture, during the period of time T, ABCDEFGHIJ form a connected area, and KLMN form also another connected area. The information is delivered in each area separately and could not be transmitted with each other. Then it is found topology structure in connected area like figure 2 .

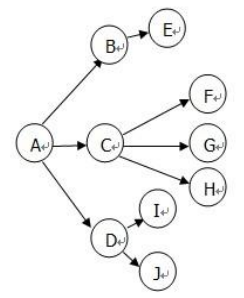

Figure 2: the topology structure in connected area

During the time of $\mathrm{T}$, corresponding topology structure is formed in areas where the information could be delivered. Assuming that node A carries information during the time of $\mathrm{T}$ and delivers the information in this area, it could be get a conclusion according to topology structure, the node A could transmit information through copy to other nodes in the connected area. Then at next time, information would be delivered to next area.

\subsection{The definition to digraph of opportunistic network}

The digraph during the time $\mathrm{T}$ could be defined according to topology structure.

Definition: the digraph of opportunistic network $\mathrm{G}=(\mathrm{V}$, E)

In which, $\mathrm{V}=\{\mathrm{A}, \mathrm{B}, \mathrm{C}, \mathrm{D}, \mathrm{E}, \mathrm{F}, \mathrm{G}, \mathrm{H}, \mathrm{I}, \mathrm{J}\}$

$\mathrm{E}=\{\langle\mathrm{A}, \mathrm{B}\rangle,\langle\mathrm{A}, \mathrm{C}\rangle,\langle\mathrm{A}, \mathrm{D}\rangle\langle\mathrm{A}, \mathrm{D}\rangle,\langle\mathrm{B}, \mathrm{E}\rangle,\langle\mathrm{C}, \mathrm{F}\rangle,\langle\mathrm{C}, \mathrm{G}\rangle,\langle\mathrm{C}$, $\mathrm{H}\rangle,\langle\mathrm{D}, \mathrm{I}\rangle,\langle\mathrm{D}, \mathrm{J}\rangle\}$

Through the digraph, the corresponding neighbor nodes of each node could be found and judged whether the surrounding nodes have communication route and could find appropriate nodes to deliver information.

3.3 Traversing the opportunistic network information transmission

According to the digraph, the network information transmission could be traversed.

$N$ stands for the node carrying information, $\mathrm{k}(i)$ stands for the neighbor node of $n$, information transmitted through nodes could be indicated as: message- $>N->\mathrm{k}(i), i>0$;

If the node $N$ carried information, according to the digraph, the node $N$ has $n$ neighbor nodes, then, it is found algorithm flowchart to show the message deliver among neighours:

It is found Pseudo-code of neighbour node ergodic algorithm in Table 1:

Table 1. Pseudo-code of neighbour node ergodic algorithm Algorithm 1 neighbour node ergodic algorithm 1 :function algorithm(message, $k(n), N$,neighbour)

2: $N<$-message

3:if( neigbour!=NULL)

4:for $i$ to $n$

$5: k(i)=$ neigbour

$6: k(i)<-N$

$7: k(i+1)<-k(i)$

$8:$ end

In this structure, it is defined $h(i)$,it means that the information is on layer $i$, if traversing layer by layer, then delivering information from layer $j$ to layer $i+1$ could indicated as:

message- $>\mathrm{h}(i)->\mathrm{h}(i+1), i>0 ;$

According to this, it is found Pseudo-code of Pseudo-code of layer delivery ergodic algorithm in Table 2:

Table 2. Pseudo-code of layer delivery ergodic algorithm

Algorithm 2 layer delivery ergodic algorithm

1:function algorithm(message, $h(n)$,node, neighbour)

2:node<-message

3:if( neigbour!=NULL)

4 :for $i 1$ to $n$

$5: h(i)<-$ node

$6: h(i+1)<-h(i)$

7 :end 
Through traversing of the information, it could find out whether information is lost or not during the information transmission in a certain period of time.

\subsection{Delivery ratio count}

In opportunistic networks, supported $t$ is reached parameter (not to consider path road). Concerning reached delivery ratio function is defined $d\left(t_{i}\right)$. These are $k$ messages delivered successful by nodes. Then during the transmission, the reached nodes of message $D(t)$ are:

$$
\begin{aligned}
D(t) & =d\left(t_{1}\right)+d\left(t_{2}\right)+\ldots \ldots+d\left(t_{k}\right) \\
& =\sum_{i=1}^{k} d\left(t_{i}\right)
\end{aligned}
$$

In opportunistic networks, each message is delivered as $d\left(s_{j}\right)$. Then the whole messages are delivered:

$$
\begin{aligned}
D(s) & =d\left(s_{1}\right)+d\left(s_{2}\right)+\ldots \ldots+d\left(s_{n}\right) \\
& =\sum_{i=1}^{n} d\left(s_{j}\right)
\end{aligned}
$$

According to (1) (2), the delivery ratio $D$ is defined:

$$
\begin{aligned}
D & =D(t) / D(s) \\
& =\sum_{i=1}^{k} d\left(t_{i}\right) / \sum_{i=1}^{n} d\left(s_{j}\right)
\end{aligned}
$$

Then according(3), it is counted delivery ratio.

\subsection{Energy consumption count}

A single node send messages and deliver must consume energy. It define that consuming energy is divided to two parts. One is send energy, anther is delivery energy.

A single node has $n$ neighbours. These are sent $n$ messages to neighbours, each neighbour need the same energy $e c$. Then a single energy $E c$ is:

$$
E c=e c+e c+\ldots \ldots+e c=\sum_{i=1}^{n} e c
$$

A single node needs the same energy ed in delivery. Then energy consumption $E d$ in delivery is:

$$
E d=e d+e d+\ldots \ldots+e d=\sum_{i=1}^{n} e d
$$

From (4), a single node needs energy consumption $E a$ is:

$$
E a=E c+E d=\sum_{i=1}^{n} e c+\sum_{i=1}^{n} e d
$$

If there are $m$ nodes taking part in corresponding in opportunistic networks. The total energy consumption $E$ in opportunistic networks is:

$$
E=m E a=m\left(\sum_{i=1}^{n} e c+\sum_{i=1}^{n} e d\right)
$$

Then according to (7), it is counted energy consumption.

\section{6 cache time count}

In opportunistic networks, cache time is taken to be the total time being used to cache all nodes during the whole life circle of a message. Supposed the cache time of a single node is $t$, these are $\mathrm{n}$ nodes taken part in delivery. Then the total cache time $T$ is:

$$
T=t_{1}+t_{2}+\ldots+t_{n}=\sum_{i=1}^{n} t_{i}
$$

According to 8, it is counted cache time.

\subsection{The priority calculation}

If the node carrying information in communication area has neighbor nodes, it means that there are communication routes between these two nodes and the value is read 1; else the node carrying information in communication area has no neighbor node, it means that there is no communication route between these two nodes and the value is read 0 . If adding one more node, the metric value will be added 1 . In this way, could we get the priority metric value of the node $P$. If $P$ is the priority metric value, the number of neighbor nodes are neighbours $(i), i>0$, then:

$$
P=\sum_{i=1}^{n} \text { neighbours }(i)
$$

According to the bigger the value of $P$, the larger amount of neighbor nodes and the more routes of information transmission, which helps to make sure the information could be delivered layer by layer.

\subsection{Algorithm flowchart}

According to the analysis, the algorithm flowchart should be as follow:

1. The node $i$ receiving information

2. Judging whether node $i$ has neighbor nodes or not

3. If there were neighbor nodes, calculating the number of neighbor nodes of node $i$

4. Looking for the neighbor node which has the most amount of neighbor nodes

5. Delivering the information to neighbor nodes

6. Saving the information

It is found Pseudo-code of PSDRA in Table 3.

Table 3. Pseudo-code of PSDRA

Algorithm 3 PSDRA Algorithm

1: function PSDRA (message , neighbors ,node)

2: node<-message

3: find neighbors

4: if(neighbors!=NULL)

5: count neighbors

6: find maximum neighbor

7: maximum neighbor<-node 


\section{Simulation experiment}

A simulation experiment is made under ONE platform. Simulate a person with intelligent Bluetooth device is walking in the city of Helsinki, as is shown in the graph, when SPMBM model is adopted and simulation indicators are preset, the shortest path between two nodes can be calculated according to the nodes and routes shown in the map by using Dijkstra algorithm. Then through the comparison between the four indicators of PSDRA and those of the other three algorithms, it is easy to find the best algorithm. Table 4 is simulation experiment.

The delivery ratio of several algorithms is shown in figure 3 .

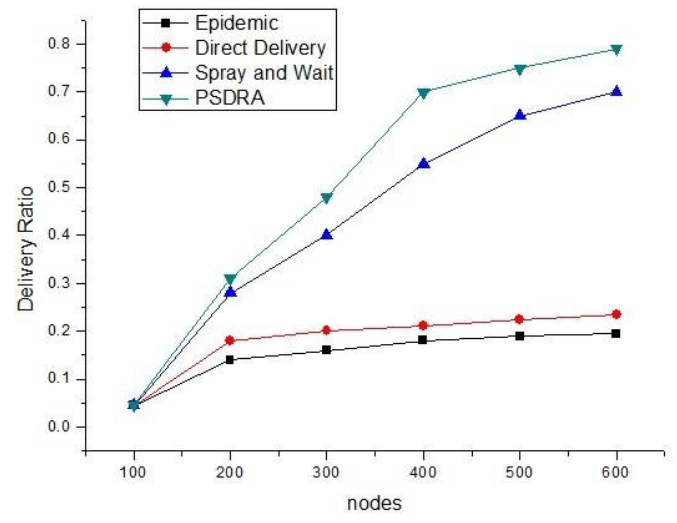

Figure 3 delivery ratio

The abscissa represents the number of nodes, while the ordinate represents the delivery ratio. The delivery ratio of different algorithms are rising with the gradual increase of nodes. From figure 3 , it is easy to find that when compared with Epidemic algorithm, PSDRA and spray and wait algorithm have a relatively high delivery ratio. Figure 4 shows the change of cache time with the gradual increase of nodes

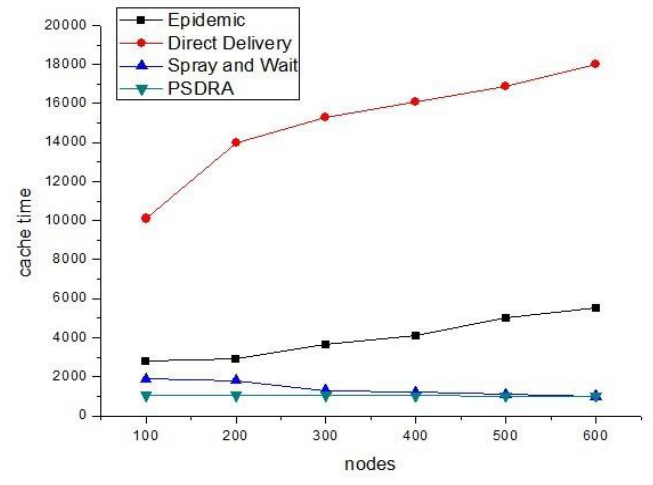

Figure 4 cache time

We can easily find that with the increase of nodes, the cache time of direct delivery algorithm rises dramatically while that of the other algorithms are not obvious. PSDRA is the best of others.

Figure 5 shows the relationship between the increase of nodes and energy consumption.

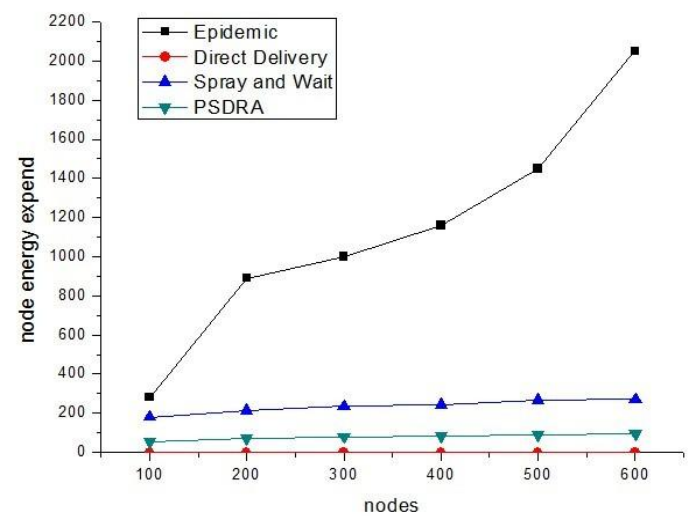

Figure 5 node energy expend

Direct delivery algorithm does not consume energy because of direct transmission. It is obvious that epidemic algorithm has the highest energy consumption, spray and wait algorithm second to it and PSDRA the least. So PSDRA has a priority over the other two algorithms in this aspect.

\section{REFERENCES}

[1] Jacquet P, Mans B, Rodolakis G. Information propagation speed in mobile and delay tolerant networks[J]. Information Theory, IEEE Transactions on, 2010, 56(10): 5001-5015.

[2] Li Y, Hui P, Jin D, et al. Evaluating the impact of social selfishness on the epidemic routing in delay tolerant networks[J] Communications Letters, IEEE, 2010, 14(11): 1026-1028.

[3] Sanchez T, Chen D T N, DeCamp S J, et al. Spontaneous motion in hierarchically assembled active matter[J]. Nature, 2012, 491(7424): 431-434.

[4] [6] Hui P, Crowcroft J, Yoneki E. Bubble rap: Social-based forwarding in delay-tolerant networks[J]. Mobile Computing, IEEE Transactions on, 2011, 10(11): 1576-1589.

[5] [7] Zhang L, Zhou XW, Wang JP, Deng Y, Wu QW. Routing protocols for delay and disruption tolerant networks. Journal of Software, 2010,21(10):2554-2572.

[6] [8]Nekovee M.Epidemic algorithms for reliable and efficient information dissemination in vehicular[J].Intelligent Transport Systems, 2009, 3 (2) : 104-110.

[7] [9] D. Karamshuk, C. Boldrini, M. Conti, and A. Passarella. Human mobility models for opportunistic networks. IEEE Comm. Mag,

[8] 46(12):157-165, Dec. 2011.

[9] [10] Gao, W. and Cao, G. 2011. User-centric data dissemination in disruption tolerant networks. In Proceedings of IEEE INFOCOM. 3119--3127.

[10] B. Burns, O. Brock, and B. Levine. Mv routing and capacity building in disruption tolerant networks. InIn Proc. IEEE Infocom, August 2010.

[11] Khouzani M H R, Eshghi S, Sarkar S, et al. Optimal energy-aware epidemic routing in DTNs[C]//Proceedings of the thirteenth ACM international symposium on Mobile Ad Hoc Networking and Computing. ACM, 2012: 175-182.

[12] Chawla M. Comparing Delay Tolerant Network Routing Protocols for Optimizing L-Copies in Spray and Wait Routing for Minimum Delay[J]. 2013. 